\title{
Control treatments in designs with split units generated by Latin squares
}

\author{
Shinji Kuriki ${ }^{1}$, Iwona $\mathrm{Mejza}^{2}$, Kazuhiro Ozawa ${ }^{3}$, Stanisław Mejza ${ }^{2}$ \\ ${ }^{1}$ Department of Mathematical Sciences, Graduate School of Engineering,Osaka Prefecture \\ University, 1-1 Gakuen-cho, Naka-ku, Sakai, Osaka 599-8531, Japan \\ ${ }^{2}$ Department of Mathematical and Statistical Methods, Poznan University of Life Sciences, \\ Wojska Polskiego 28, PL-60-637 Poznań, Poland \\ ${ }^{3}$ Department of Nursing, Gifu College of Nursing, Hashima, Gifu 501-6295, Japan
}

\section{SUMMARY}

\begin{abstract}
This paper deals with two-factor experiments with split units. The whole plot treatments occur in a repeated Latin square, modified Latin square or Youden square, while subplot treatments occur in a block design within the whole plots. The statistical properties of the considered designs are examined. Special attention is paid to the case where one of the treatments is an individual control or an individual standard treatment. In addition, we give a brief overview of work on the design of experiments using the considered designs, as well as possible arrangements of controls in the experiments.
\end{abstract}

Key words: Latin square, Youden square, split units, efficiency factors, merging treatment method.

\section{Introduction}

In agricultural field experiments, a row-column design or repeated row-column design is quite often used to eliminate real or potential orthogonally disposed heterogeneity of the experimental material. From a statistical point of view, a Latin square is then the appropriate design. This design is especially efficient in agricultural field experiments. It possesses many desirable and optimal statistical properties. In the Latin square every treatment occurs once in each row and once in each column. This means that the component designs, both with respect to the rows and with respect to the columns, are randomized complete blocks. Another row-column design with desirable statistical 
properties is the Youden square. In this design the treatments occur in randomized complete blocks with respect to rows (or columns), while with respect to columns (or rows) they occur in a symmetrical balanced incomplete block design.

In the present work, whole plots occur in one of the above designs or in some modification of them. Then the whole plots are divided into subplots as in the usual split-plot design. Hence the final design is called a split-plot design generated by a Latin square. This means that the row-column structure (Latin square, modified Latin square or Youden square) is repeated over the superblocks. The arrangement of treatments may be the same or different in each superblock.

Let us consider two-factor experiments in which the first factor (or set of factors) $A$ occurs at $a$ levels $A_{1}, A_{2}, \ldots, A_{a}$, and the second factor (or set of factors) $B$ occurs at $t$ levels $B_{1}, B_{2}, \ldots, B_{t}$.

We consider here a situation where the levels of factor $A$ are arranged on plots (whole plots) in a (modified) Latin square, while the levels of the second factor are arranged within the whole plots on the subplots. The levels of factor $A$ will then be called whole plot treatments, while the levels of factor $B$ will be called subplot treatments.

The aim of this paper is to propose a new design, as well as giving a survey of existing methods of constructing a new design in which the whole plot treatments occur in a Latin square or in certain modifications thereof, while subplots occur on subplots within whole plots. Special attention is paid to the use of control treatments in such designs. The subplot treatments will occur in any proper design.

Let us assume that the experimental units are divided into $R$ superblocks, and each superblock is divided into $q p$ whole plot units which form a lattice with $q$ rows and $p$ columns. Additionally let each of the whole plots be divided into $k$ subplots. In the row-column design we can consider two component block designs, one of them with respect to rows, and the - other with respect to columns. 
The arrangement of the whole plot and subplot treatments on the experimental material is based on a proper scheme of randomizations (cf. Kachlicka and Mejza, 1996). This scheme includes randomizations of the superblocks, the rows (columns), the columns (rows) and the subplots. As a result of such randomizations and some additional assumptions, we can express the observations by a linear mixed model with random superblock, row and column effects and fixed treatment combination effects. The applied scheme of randomizations and the structure of the experimental material lead to a linear model of observations, possessing an orthogonal block structure. Then the overall analysis can be split into so-called strata, as in multistratum experiments. In our case we have five strata, i.e. the inter-superblock stratum (I), the inter-row stratum (II), the inter-column stratum (III), the inter-whole plot stratum (IV) and finally the inter-subplot stratum (V).

In Kachlicka and Mejza (1996) a method was given for analyzing a twofactor experiment carried out in a design in which whole plot treatments occur in repeated row-column designs while the subplot treatments occur in complete or incomplete block designs where whole plots are treated as blocks. However, we continue to observe a lack of construction methods for the considered class of designs. The present work constitutes an important supplement to the aforementioned paper. In particular the papers by Kachlicka and Mejza (2003), Kachlicka et al. (2004), Kuriki et al. (2009) and Mejza et al. (2009) examine the statistical properties of a design in which whole plot treatments occur in a Youden square and subplot treatments occur in a balanced incomplete block design (BIBD) or in group-divisible partially balanced incomplete block designs with two efficiency classes (GDPBIBD(2)s). The BIBD and GDPBIBD(2)s are very useful in biological and agricultural experiments, and hence they are often used to generate new more complex designs with split units (cf. Mejza and Mejza, 1996, Hering and Mejza, 2002, Kachlicka and Mejza, 2006, Mejza and Kuriki, 2013).

The treatment combinations are considered as treatments with the natural lexicographical order of combinations $A_{s} B_{h}(s=1,2, \ldots, a ; h=1,2, \ldots, t)$ and 
the usual expression of the treatment effect as the sum of the factor effects and the interaction effects. Let $v=a t$ denote the number of treatments.

The statistical properties of the design are connected with the algebraic properties of the so-called information matrices $\mathbf{A}_{f}, f=1,2,3,4,5$ which in the design considered here have the forms:

$$
\begin{aligned}
& \mathbf{A}_{1}=(R p q k)^{-1}\left(R \mathbf{N}_{0} \mathbf{N}_{0}^{\prime}-\mathbf{r} \mathbf{r}^{\prime}\right), \\
& \mathbf{A}_{2}=(p q k)^{-1}\left(p \mathbf{N}_{1} \mathbf{N}_{1}^{\prime}-\mathbf{N}_{0} \mathbf{N}_{0}^{\prime}\right), \\
& \mathbf{A}_{3}=(p q k)^{-1}\left(q \mathbf{N}_{2} \mathbf{N}_{2}^{\prime}-\mathbf{N}_{0} \mathbf{N}_{0}^{\prime}\right), \\
& \mathbf{A}_{4}=(p q k)^{-1}\left(\mathbf{N}_{0} \mathbf{N}_{0}^{\prime}+p q \mathbf{N}_{3} \mathbf{N}_{3}^{\prime}-q \mathbf{N}_{2} \mathbf{N}_{2}^{\prime}-p \mathbf{N}_{1} \mathbf{N}_{1}^{\prime}\right), \\
& \mathbf{A}_{5}=\mathbf{r}^{\delta}-k^{-1} \mathbf{N}_{3} \mathbf{N}_{3}^{\prime},
\end{aligned}
$$

where $\mathbf{N}_{0}, \mathbf{N}_{1}, \mathbf{N}_{2}, \mathbf{N}_{3}$ are the incidence matrices of treatments vs. superblocks, treatments vs. rows, treatments vs. columns and treatments vs. whole plots respectively, $\mathbf{r}$ denotes the vector of treatment replicates, and $\mathbf{r}^{\delta}$ stands for the diagonal matrix with diagonal elements equal to the numbers of treatment replicates.

A desirable statistical property which we shall be examining is called the general balance (cf. Bailey, 1995). A design is generally balanced iff the information matrices satisfy the conditions (cf. Mejza, 1992):

$$
\mathbf{A}_{f} \mathbf{r}^{-\delta} \mathbf{A}_{f^{\prime}}=\mathbf{A}_{f^{\prime}} \mathbf{r}^{-\delta} \mathbf{A}_{f}, \quad f \neq f^{\prime}, f, f^{\prime}=1,2,3,4,5 .
$$

Let us assume that the above commutativities of the information matrices hold, and hence the considered designs are generally balanced.

Let $\mathbf{p}_{j}$ define contrasts of the form $\mathbf{p}_{j}^{\prime} \boldsymbol{\tau}, j=1,2, \ldots, v-1$, where the vectors $\mathbf{p}_{j}$ are connected with eigenvectors of information matrices, and $\boldsymbol{\tau}$ denotes the vector of effects of treatment combinations. Then the $\mathbf{p}_{j}^{\prime} \boldsymbol{\tau}$ are called the basic contrasts (cf. Pearce et al., 1974). Next, the eigenvalues of the information matrices can be identified as stratum efficiency factors of the design with respect to the $j$-th basic contrast in the $f$-th stratum, $f=1,2,3,4,5 ; j=1,2, \ldots, v-1$ (cf. Houtman and Speed, 1983). 
The stratum efficiency factors measure the amount of information that is included in the strata for estimating the treatment effect contrast. A stratum efficiency factor equal to 1 means that the particular contrast is estimable with full efficiency only in that stratum. A stratum efficiency factor equal to 0 means that the contrast is not estimable in that stratum (it is confounded). The sum of stratum efficiency factors concerning each treatment contrast is equal to 1 .

We shall assume throughout that the contents of whole plots within each superblock are all the same with respect to subplot treatments. This means that the arrangements of whole plot and subplot treatments can be expressed by the Kronecker product of proper incidence matrices.

\section{Whole plot design}

\subsection{Latin square}

Let $\mathbf{N}_{A}=\mathbf{J}_{a}$ denote the treatments vs. rows and treatments vs. columns incidence matrix in a Latin square, while $\mathbf{N}_{B}$ denotes the incidence matrix for the subplot treatments. The vectors $\mathbf{r}_{A}=a \mathbf{1}_{a}$ and $\mathbf{r}_{B}$ denote the appropriate vectors of whole plot and subplot treatment replicates, where $\mathbf{1}_{a}$ denotes the $a \times 1$ vector of ones and $\mathbf{J}_{a}=\mathbf{1}_{a} \mathbf{1}_{a}^{\prime}$.

Let the whole plot treatments be arranged in a Latin square, and the subplot treatments in a completely randomized design. From (1) it is easy to check that the efficiency factors of the final design for the estimation of treatment contrast effects are as in Table 1.

Table 1. Stratum efficiency factors of Latin square with split units

\begin{tabular}{ccccccc}
\hline \multirow{2}{*}{ Type of contrasts } & \multirow{2}{*}{ Number of contrasts } & \multicolumn{5}{c}{ Strata } \\
\cline { 3 - 7 } & $a-1$ & 0 & II & III & IV & V \\
\hline$A$ & $t-1$ & 0 & 0 & 0 & 0 & 1 \\
$B$ & $(a-1)(t-1)$ & 0 & 0 & 0 & 0 & 1
\end{tabular}

From Table 1 we can infer that all contrasts among the whole plot treatment effects are estimated with full efficiency in the fourth stratum. The contrasts 
among the subplot treatment effects and the interaction effects are estimated with full efficiency in the fifth stratum.

The Latin square will be used as a starting point design for further constructions. We can construct new designs using a treatment merging method (cf. Ceranka and Mejza, 1987). Moreover, by this method it is possible to construct desirable designs for subplot treatments also. Merging of two treatments leads to greater precision in the rest of the design (cf. Caliński and Kageyama, 2003).

For example, let $h=g+s$ denote the number of treatments. We perform the treatment merging method by multiplying the incidence matrix by a $(g+1) \times h$ merging matrix of the form:

$$
\mathbf{P}=\left[\begin{array}{rr}
\mathbf{I}_{g} & \mathbf{0} \\
\mathbf{0}^{\prime} & \mathbf{1}_{s}^{\prime}
\end{array}\right] .
$$

The number $s$ describes how many treatments we would like to merge into one new treatment (cf. Caliński and Kageyama, 2003). Then the number of treatments is equal to $g+1$.

Here $\mathbf{N}_{B}$ will denote the incidence matrix for the subplot treatments. Let us merge the last $s$ whole plot treatments into a new treatment. This means that together we have $a=g+1 \quad(g=a-s)$ whole plot treatments in the final design. The new incidence matrix has the form $\mathbf{M}=\mathbf{P} \mathbf{N}_{A}$.

Then we have: $\quad \mathbf{N}_{0} \mathbf{N}_{0}^{\prime}=a^{2} \mathbf{D} \otimes \mathbf{N}_{B} \mathbf{N}_{B}^{\prime}, \quad \mathbf{N}_{1} \mathbf{N}_{1}^{\prime}=\mathbf{N}_{2} \mathbf{N}_{2}^{\prime}=a \mathbf{D} \otimes \mathbf{N}_{B} \mathbf{N}_{B}^{\prime}$, $\mathbf{N}_{3} \mathbf{N}_{3}^{\prime}=a \mathbf{D}_{1} \otimes \mathbf{N}_{B} \mathbf{N}_{B}^{\prime}$ where

$$
\begin{aligned}
& \mathbf{D}=\left[\begin{array}{ll}
\mathbf{J}_{g} & s \mathbf{1} \\
s \mathbf{1}^{\prime} & s^{2}
\end{array}\right], \mathbf{D}_{1}=\left[\begin{array}{cc}
\mathbf{I}_{g} & \mathbf{0} \\
\mathbf{0}^{\prime} & s
\end{array}\right], \quad \mathbf{r}=a\left[\begin{array}{c}
\mathbf{1}_{g} \\
s
\end{array}\right] \otimes \mathbf{r}_{B}, \\
& \mathbf{r}_{B}=\mathbf{N}_{B} \mathbf{1}, \quad \mathbf{r r}^{\prime}=a^{2} \mathbf{D} \otimes \mathbf{r}_{B} \mathbf{r}_{B}^{\prime}
\end{aligned}
$$

and $\otimes$ denotes the Kronecker product of matrices. The information matrices are as follows:

$$
\begin{aligned}
& \mathbf{A}_{1}=k^{-1} \mathbf{D} \otimes\left(\mathbf{N}_{B} \mathbf{N}_{B}^{\prime}-R^{-1} \mathbf{r}_{B} \mathbf{r}_{B}^{\prime}\right), \\
& \mathbf{A}_{2}=\mathbf{A}_{3}=\mathbf{0},
\end{aligned}
$$




$$
\begin{aligned}
& \mathbf{A}_{4}=a k^{-1}\left[\left(\mathbf{D}_{1}-a^{-1} \mathbf{D}\right) \otimes \mathbf{N}_{B} \mathbf{N}_{B}^{\prime}\right], \\
& \mathbf{A}_{5}=a \mathbf{D}_{1} \otimes\left(\mathbf{r}_{B}^{\delta}-k^{-1} \mathbf{N}_{B} \mathbf{N}_{B}^{\prime}\right) .
\end{aligned}
$$

From (2) we can infer that all contrasts among the whole plot treatments are estimated with full efficiency in the fourth stratum. The contrasts among the subplot treatments are estimated in the first and the fifth stratum, while the contrasts among the interaction effects are estimated with efficiencies depending on the efficiencies of the subplot design.

Finally, let us note that the treatments being merged can be regarded as the control treatment or standard treatment, which is replicated more times than other treatments. This is also one of the possible ways to incorporate a control treatment into a design.

\subsection{Youden square}

Le us assume that each superblock has a Youden square structure with $q$ rows and $a$ columns. Moreover, the subdesign of the Youden square with respect to columns is a symmetrical balanced incomplete block design (BIBD) with parameters as follows: $\operatorname{BIBD}\left(v_{A}, b_{A}, r_{A}, k_{A}, \lambda_{A}\right)$. Then the following relationships hold:

$$
k_{A}=r_{A}=q, \quad b_{A}=v_{A}=a \quad \text { and } \quad \lambda_{A}=\frac{q(q-1)}{a-1}
$$

Let $\mathbf{N}_{A}$ be the whole plot treatments vs. columns incidence matrix in the Youden square. Then the so-called $\mathbf{C}$-matrix for the Youden square subdesign with respect to columns is equal to $\mathbf{C}_{A}=r_{A} \mathbf{I}-k_{A}^{-1} \mathbf{N}_{A} \mathbf{N}_{A}^{\prime}$. The so-called efficiency factor connected with this matrix is equal to $\varepsilon_{A}=a(q-1) / q(a-1)$, while $\rho_{A}=a-1$ is its multiplicity (cf. Caliński and Kageyama, 2000).

Let $D_{B}\left(t, b, k, \mathbf{N}_{B}\right)$ denote any proper block design in which $t$ subplot treatments occur in $b$ blocks of size $k$ according to the incidence matrix $\mathbf{N}_{B}$, and let $\mathbf{r}_{B}$ be the vector of treatment replicates $\left(\mathbf{r}_{B}=\mathbf{N}_{B} \mathbf{1}_{n_{B}}\right)$, where $\mathbf{1}_{n_{B}}$ stands for the vector of ones, and $n_{B}=b k$. 
Let $\mathbf{C}_{B}=\mathbf{r}_{B}^{\delta}-k^{-1} \mathbf{N}_{B} \mathbf{N}_{B}^{\prime}$ be the $\mathbf{C}$-matrix of the block design $D_{B}\left(t, b, k, \mathbf{N}_{B}\right)$ and let $\xi_{h}$ be an eigenvalue of the matrix $\mathbf{C}_{B}$ corresponding to an eigenvector $\mathbf{c}_{h}$ with respect to $\mathbf{r}_{B}^{\delta}$, i.e. let $\mathbf{C}_{B} \mathbf{c}_{h}=\xi_{h} \mathbf{r}_{B}^{\delta} \mathbf{c}_{h}, h=1,2, \ldots, t$. The eigenvectors can be chosen to be $\mathbf{r}_{B}^{\delta}$ - orthonormal in pairs, i.e., $\mathbf{c}_{i}^{\prime} \mathbf{r}_{B}^{\delta} \mathbf{c}_{i}=1$ and $\mathbf{c}_{i}^{\prime} \mathbf{r}_{B}^{\delta} \mathbf{c}_{h}=0$, for $i \neq h ; i, h=1,2, \ldots, t$. Since $\mathbf{C}_{B} \mathbf{1}_{t}=\mathbf{0}$, the last eigenvector may be chosen as $\mathbf{c}_{t}=n_{B}^{-1 / 2} \mathbf{1}_{t}$.

The statistical properties of the design are connected with the algebraic properties of the information matrices $\mathbf{A}_{f}, f=1,2,3,4,5$, which in the design considered here have the forms:

$$
\begin{aligned}
& \mathbf{A}_{1}=(R q a k)^{-1}\left(R \mathbf{N}_{0} \mathbf{N}_{0}^{\prime}-\mathbf{r} \mathbf{r}^{\prime}\right)=\frac{q}{a} \mathbf{J}_{a} \otimes\left(b^{-1} \mathbf{N}_{B} \mathbf{N}_{B}^{\prime}-n_{B}^{-1} \mathbf{r}_{B} \mathbf{r}_{B}^{\prime}\right), \\
& \mathbf{A}_{2}=(q a k)^{-1}\left(q \mathbf{N}_{1} \mathbf{N}_{1}^{\prime}-\mathbf{N}_{0} \mathbf{N}_{0}^{\prime}\right)=0, \\
& \mathbf{A}_{3}=(q a k)^{-1}\left(a \mathbf{N}_{2} \mathbf{N}_{2}^{\prime}-\mathbf{N}_{0} \mathbf{N}_{0}^{\prime}\right)=\frac{a-q}{(a-1) k}\left(\mathbf{I}_{a}-\frac{1}{a} \mathbf{J}_{a}\right) \otimes \mathbf{N}_{B} \mathbf{N}_{B}^{\prime}, \\
& \mathbf{A}_{4}=(q a k)^{-1}\left(\mathbf{N}_{0} \mathbf{N}_{0}^{\prime}+q a \mathbf{N}_{3} \mathbf{N}_{3}^{\prime}-a \mathbf{N}_{2} \mathbf{N}_{2}^{\prime}-q \mathbf{N}_{1} \mathbf{N}_{1}^{\prime}\right)= \\
& =\frac{a(q-1)}{(a-1) k}\left(\mathbf{I}_{a}-\frac{1}{a} \mathbf{J}_{a}\right) \otimes \mathbf{N}_{B} \mathbf{N}_{B}^{\prime}, \\
& \mathbf{A}_{5}=\mathbf{r}^{\delta}-k^{-1} \mathbf{N}_{3} \mathbf{N}_{3}^{\prime}=q \mathbf{I}_{a} \otimes\left(\mathbf{r}_{B}^{\delta} \mathbf{I}_{b}-\frac{1}{k} \mathbf{N}_{B} \mathbf{N}_{B}^{\prime}\right), \\
& \text { where } \quad \mathbf{r r}^{\prime}=q^{2} \mathbf{J}_{a} \otimes \mathbf{r}_{B} \mathbf{r}_{B}^{\prime}, \quad \mathbf{r}^{\delta}=q \mathbf{I}_{a} \otimes \mathbf{r}_{B}^{\delta}, \quad \mathbf{N}_{0} \mathbf{N}_{0}^{\prime}=q^{2} \mathbf{J}_{a} \otimes \mathbf{N}_{B} \mathbf{N}_{B}^{\prime}, \\
& \mathbf{N}_{1} \mathbf{N}_{1}^{\prime}=q \mathbf{J}_{a} \otimes \mathbf{N}_{B} \mathbf{N}_{B}^{\prime}, \\
& \mathbf{N}_{2} \mathbf{N}_{2}^{\prime}=\mathbf{N}_{A} \mathbf{N}_{A}^{\prime} \otimes \mathbf{N}_{B} \mathbf{N}_{B}^{\prime}, \\
& \mathbf{N}_{3} \mathbf{N}_{3}^{\prime}=q \mathbf{I}_{a} \otimes \mathbf{N}_{B} \mathbf{N}_{B}^{\prime}, \\
& \mathbf{N}_{A} \mathbf{N}_{A}^{\prime}=\left(r_{A}-\lambda_{A}\right) \mathbf{I}_{a}+\lambda_{A} \mathbf{J}_{a}=\frac{q(a-q)}{a-1} \mathbf{I}_{a}+\frac{q(q-1)}{a-1} \mathbf{J}_{a} .
\end{aligned}
$$

The matrices $\mathbf{N}_{0}, \mathbf{N}_{1}, \mathbf{N}_{2}, \mathbf{N}_{3}$ are the incidence matrices of treatments vs. superblocks, treatments vs. rows, treatments vs. columns and treatments vs. whole plots respectively, $\mathbf{r}$ denotes the vector of treatment replicates, and $\mathbf{r}^{\delta}$ 
stands for the diagonal matrix with diagonal elements equal to the numbers of treatment replicates.

From (3) we can infer that the contrasts among the whole plot treatment effects are estimated in the third and fourth strata with efficiencies $1-\varepsilon_{A}$ and $\varepsilon_{A}$ respectively. The contrasts among the subplot treatment effects are estimated in the first and fifth strata, with efficiencies depending on the design used for subplot treatments. Similarly, contrasts among the interaction effects can be estimated in the third, fourth and/or fifth strata. All of these remarks are summarized in Table 2 (cf. Kuriki et al., 2009). The stratum efficiency factors are functions of $\varepsilon_{A}$ and $\xi_{h}, h=1,2, \ldots, t-1$.

Table 2. Stratum efficiency factors of the design under consideration

\begin{tabular}{ccrrcccc}
\hline \multirow{2}{*}{ Type of contrasts } & $\begin{array}{c}\text { Number of } \\
\text { contrasts }\end{array}$ & I & II & III & IV & V \\
\hline$A$ & $a-1$ & 0 & 0 & $1-\varepsilon_{A}$ & $\varepsilon_{A}$ & 0 \\
$B_{h}$ & & & $1-\xi_{h}$ & 0 & 0 & 0 & $\xi_{h}$ \\
$h=1,2, \ldots, t-1$ & 1 & & & & & \\
$(A \times B)_{h}$ & $a-1$ & 0 & 0 & $\left(1-\varepsilon_{A}\right)\left(1-\xi_{h}\right)$ & $\varepsilon_{A}\left(1-\xi_{h}\right)$ & $\xi_{h}$ \\
$h=1,2, \ldots, t-1$ & & & & &
\end{tabular}

\section{Subplot designs}

\subsection{Subplot treatments in BIBD}

In this section we will consider two particular cases of optimal designs for subplot treatments.

Let the whole plot treatment be arranged in a Youden square as in Section 2.2, while subplot treatments are arranged in a balanced incomplete block design (BIBD) with the parameters $v_{B}=t, b_{B}, r_{B}, k_{B}, \lambda_{B}, R=b_{B}, k=k_{B}$. 
Let $\mathbf{C}_{B}$ be the $\mathbf{C}$-matrix of the BIBD for subplot treatments, and let $\varepsilon_{B}=t(k-1) / k(t-1)$ denote the efficiency factors, with multiciplities $\rho_{B}=t-1$.

The final design is generally balanced, and the efficiency factors are presented in Table 3 (cf. Kachlicka and Mejza, 2004).

Table 3. Stratum efficiency factors of the design under consideration

\begin{tabular}{ccccccc}
\hline Type of & Number of & \multicolumn{5}{c}{ Strata } \\
\cline { 3 - 7 } contrasts & contrasts & I & II & III & IV & V \\
\hline$A$ & $a-1$ & 0 & 0 & $1-\varepsilon_{A}$ & $\varepsilon_{A}$ & 0 \\
$B$ & $t-1$ & $1-\varepsilon_{B}$ & 0 & 0 & 0 & $\varepsilon_{B}$ \\
$A \times B$ & $(a-1)(t-1)$ & 0 & 0 & $\left(1-\varepsilon_{A}\right)\left(1-\varepsilon_{B}\right)$ & $\varepsilon_{A}\left(1-\varepsilon_{B}\right)$ & $\varepsilon_{B}$ \\
\hline
\end{tabular}

\subsection{Subplot treatments in GDPBIBD(2)}

Let the whole plot treatments be arranged in a Youden square, while the subplot treatments are arranged in a group-divisible (GD) partially balanced incomplete block design with two association classes (GDPBIBD(2)) (cf. Clatworthy, 1973). The parameters of the $\operatorname{GDPBIBD}(2)$ for subplot treatments are as follows: $v_{B}=t=m \cdot n, b_{B}, r_{B}, k_{B}(=k), \lambda_{B 1}, \lambda_{B 2}$. The parameters $\lambda_{B 1}, \lambda_{B 2}$ respecttively denote the numbers of occurring pairs of treatments from the same group and different groups in the blocks. The concurrence matrix $\mathbf{N}_{B} \mathbf{N}_{B}^{\prime}$ has three eigenvalues $\omega_{i}$ with multiplicities $\rho_{i}$, where

$$
\begin{aligned}
& \omega_{0}=r_{B} k_{B}, \quad \omega_{1}=r_{B}-\lambda_{B 1}, \quad \omega_{2}=r_{B} k_{B}-v_{B} \lambda_{B 2}, \\
& \rho_{0}=1, \quad \rho_{1}=m(n-1), \quad \rho_{2}=m-1 .
\end{aligned}
$$

Let $\mathbf{C}_{B}$ denote the $\mathbf{C}$-matrix of the $\operatorname{GDPBIBD}(2)$ for subplot treatments, and let $\varepsilon_{B i}=1-\omega_{i} / r_{B} k_{B}$ denote the efficiency factors, with multiplicities $\rho_{B i}, \quad i=0,1,2$, where $\sum_{i=0}^{2} \rho_{B i}=v_{B}$. The overall statistical properties (estimability of treatment effect contrasts and their efficiencies) of the final design (Table 4) are connected with these efficiency factors (cf. Mejza et al., 
2009). The ranks of the information matrices $\mathbf{A}_{i}, i=1,2,3,4,5$, depend on the type of GDPBIBD(2) applied.

Table 4. Stratum efficiency factors of the design under consideration

\begin{tabular}{ccccccc}
\hline \multirow{2}{*}{$\begin{array}{c}\text { Type of } \\
\text { contrasts }\end{array}$} & $\begin{array}{c}\text { Number of } \\
\text { contrasts }\end{array}$ & I & II & III & IV & V \\
\cline { 3 - 7 }$A$ & $a-1$ & 0 & 0 & $1-\varepsilon_{A}$ & $\varepsilon_{A}$ & 0 \\
$B^{(1)}$ & $\rho_{B 1}$ & $1-\varepsilon_{B 1}$ & 0 & 0 & 0 & $\varepsilon_{B 1}$ \\
$B^{(2)}$ & $\rho_{B 2}$ & $1-\varepsilon_{B 2}$ & 0 & 0 & 0 & $\varepsilon_{B 2}$ \\
$A \times B^{(1)}$ & $(a-1) \rho_{B 1}$ & 0 & 0 & $\left(1-\varepsilon_{A}\right)\left(1-\varepsilon_{B 1}\right)$ & $\varepsilon_{A}\left(1-\varepsilon_{B 1}\right)$ & $\varepsilon_{B 1}$ \\
$A \times B^{(2)}$ & $(a-1) \rho_{B 2}$ & 0 & 0 & $\left(1-\varepsilon_{A}\right)\left(1-\varepsilon_{B 2}\right)$ & $\varepsilon_{A}\left(1-\varepsilon_{B 2}\right)$ & $\varepsilon_{B 2}$ \\
\hline
\end{tabular}

\section{Control treatments}

In the natural sciences, experiments are performed to compare known treatments (also called test treatments) with a set of control treatments or standards. Here we consider mainly a situation in which we would like to compare whole plot treatments with some controls (whole plot controls). One of the possible ways to arrange control treatments in a Latin square is to use a merging method, as described in Section 2.1. Another way is to supplement the Youden square by additional control treatments. This can be expressed by the following incidence matrix:

$$
\mathbf{N}_{A}^{*}=\left[\begin{array}{c}
\mathbf{N}_{A} \\
\mathbf{J}_{s \times b_{A}}
\end{array}\right] .
$$

More exactly, $a$ test treatments of the factor $A$ are assigned in the $(q \times a)$ Youden square, and additionally $s$ control treatments are added.

Using the previous characterization of the Youden square with the whole plot test treatments vs. columns incidence matrix $\mathbf{N}_{A}$ we have

$$
k_{A}=r_{A}=q, p=b_{A}=v_{A}=a, \lambda_{A}=\frac{q(q-1)}{a-1}, \varepsilon_{A}=\frac{a(q-1)}{q(a-1)},
$$


with multiplicity $\rho_{A}=a-1$.

The final design with respect to the whole plot treatments has the following parameters:

$$
\begin{aligned}
& v_{A}^{*}=a+s, \quad k_{A}^{*}=q+s, \quad b_{A}^{*}=b_{A}, \quad \mathbf{r}_{A}^{*}=\left[q \mathbf{1}_{a}^{\prime} \vdots a \mathbf{1}_{s}^{\prime}\right]^{\prime} \\
& \varepsilon_{A 0}^{*}=1, \quad \rho_{A 0}^{*}=s, \quad \varepsilon_{A 1}^{*}=1-\frac{k_{A}\left(1-\varepsilon_{A}\right)}{k_{A}+s}, \quad \rho_{A 1}^{*}=a-1
\end{aligned} .
$$

Similarly, using the characterization of subplot treatments occurring in the balanced incomplete block design (BIBD) with the parameters $v_{B}=t, b_{B}, r_{B}, k_{B}, \lambda_{B}$ and incidence matrix $\mathbf{N}_{\mathrm{B}}$, we have the following parameters of the design with respect to subplot treatments:

$$
R=b_{B}, q=a+s, k=k_{B}, \lambda_{B}=\frac{r_{B}(k-1)}{t-1}, \varepsilon_{B}=\frac{t(k-1)}{k(t-1)},
$$

with multiplicity $\rho_{B}=t-1$.

Finally, the stratum efficiency factors of that design are presented in Table 5 (cf. Mejza and Kuriki, 2013).

In Table 5 the abbreviation $A^{(1)}$ represents the set of contrasts among effects of whole plot test treatments only; $A^{(2)}$ represents the set of contrasts among effects of whole plot control treatments only; and $A^{(3)}$ represents the contrast among the effects of the whole plot test and control treatments.

Table 5. Stratum efficiency factors of the design under consideration

\begin{tabular}{ccccccc}
\hline Type of & No of & \multicolumn{5}{c}{ Strata } \\
\cline { 3 - 7 } contrasts & contrasts & I & II & III & IV & V \\
\hline$A^{(1)}$ & $a-1$ & 0 & 0 & $1-\varepsilon_{A 1}^{*}$ & $\varepsilon_{A 1}^{*}$ & 0 \\
$A^{(2)}$ & $s-1$ & 0 & 1 & 0 & 0 & 0 \\
$A^{(3)}$ & 1 & 0 & 1 & 0 & 0 & 0 \\
$B$ & $t-1$ & $1-\varepsilon_{B}$ & 0 & 0 & 0 & $\varepsilon_{B}$ \\
$A^{(1)} \times B$ & $(a-1)(t-1)$ & 0 & 0 & $\left(1-\varepsilon_{A 1}^{*}\right)\left(1-\varepsilon_{B}\right)$ & $\varepsilon_{A 1}^{*}\left(1-\varepsilon_{B}\right)$ & $\varepsilon_{B}$ \\
$A^{(2)} \times B$ & $(s-1)(t-1)$ & 0 & $1-\varepsilon_{B}$ & 0 & 0 & $\varepsilon_{B}$ \\
$A^{(3)} \times B$ & $t-1$ & 0 & $1-\varepsilon_{B}$ & 0 & 0 & $\varepsilon_{B}$ \\
\hline
\end{tabular}




\section{Application}

Let us consider a two-factor experiment in which we have four whole plot treatments $(a=4)$ and three subplot treatments $(t=3)$. The experiment was set up in four superblocks $(R=4)$, each divided into four rows $(q=4)$ and four columns $(a=4)$. Additionally, each whole plot is divided into three subplots ( $k$ $=3$ ) to accommodate the subplot treatments. A possible arrangement of whole plot treatments in a Latin square could be the following:

\begin{tabular}{|l|l|l|l|}
\hline$A_{2}$ & $A_{4}$ & $A_{3}$ & $A_{1}$ \\
\hline$A_{3}$ & $A_{1}$ & $A_{4}$ & $A_{2}$ \\
\hline$A_{4}$ & $A_{2}$ & $A_{1}$ & $A_{3}$ \\
\hline$A_{1}$ & $A_{3}$ & $A_{2}$ & $A_{4}$ \\
\hline
\end{tabular}

The incidence matrix is as follows:

$$
\mathbf{N}_{A}=\left[\begin{array}{llll}
1 & 1 & 1 & 1 \\
1 & 1 & 1 & 1 \\
1 & 1 & 1 & 1 \\
1 & 1 & 1 & 1
\end{array}\right] .
$$

Let us merge the last two whole plot treatments. This we can do by renumbering $A_{4}$ into $A_{3}$ or multiplying the incidence matrix $\mathbf{N}_{A}$ by the matrix $\mathbf{P}$

$$
\mathbf{P}=\left[\begin{array}{llll}
1 & 0 & 0 & 0 \\
0 & 1 & 0 & 0 \\
0 & 0 & 1 & 1
\end{array}\right] .
$$

Then we have

$$
\mathbf{P N}_{A}=\left[\begin{array}{llll}
1 & 1 & 1 & 1 \\
1 & 1 & 1 & 1 \\
2 & 2 & 2 & 2
\end{array}\right] .
$$


The same merging matrix can be used, for example, to construct a subdesign for the subplot treatments. Let the starting point design be the symmetric BIB design for four treatments with incidence matrix

$$
\mathbf{N}_{B}=\left[\begin{array}{llll}
0 & 1 & 1 & 1 \\
1 & 1 & 0 & 1 \\
1 & 1 & 1 & 1 \\
1 & 0 & 1 & 0
\end{array}\right] .
$$

After merging the last two treatments in $\mathbf{N}_{B}$ the subplot treatments occur in an efficiency balanced block design with unequal numbers of replicates (cf. Caliński and Kageyama, 2003). The arrangement of the subplot treatments in superblocks can be represented schematically as follows:

\begin{tabular}{|l|l|l|l|}
\hline$B_{2}$ & $B_{1}$ & $B_{1}$ & $B_{1}$ \\
\hline$B_{3}$ & $B_{2}$ & $B_{3}$ & $B_{2}$ \\
\hline$B_{3}$ & $B_{3}$ & $B_{3}$ & $B_{3}$ \\
\hline
\end{tabular}

In each superblock the subplot treatments are arranged on the subplots within the whole plots according to the incidence matrix

$$
\mathbf{P N}_{B}=\left[\begin{array}{llll}
0 & 1 & 1 & 1 \\
1 & 1 & 0 & 1 \\
2 & 1 & 2 & 1
\end{array}\right] .
$$

Hence we have: $v_{B}=b=3, R=b_{B}=4, k=3, \mathbf{r}_{B}=[3,3,6]^{\prime}, \xi_{h}=8 / 9, h=1,2$, $\xi_{3}=0$.

In Figure 1 the arrangement of the subplot and the whole plot treatments in the superblocks is given. The stratum efficiency factors of the design are given in Table 6.

Here, $A^{(1)}$ and $A^{(2)}$ denote respectively the basic contrast between the main effects of whole plot test treatments and the basic contrast between the main effects of whole plot test and control treatments. $B^{(1)}$ and $B^{(2)}$ denote the same 
Superblock I

\begin{tabular}{|c|c|c|c|c|c|c|c|c|c|c|c|}
\hline \multicolumn{3}{|c|}{$A_{2}$} & \multicolumn{3}{|c|}{$A_{3}$} & \multicolumn{3}{|c|}{$A_{3}$} & \multicolumn{3}{|c|}{$A_{1}$} \\
\hline$B_{2}$ & $B_{3}$ & $B_{3}$ & $B_{2}$ & $B_{3}$ & $B_{3}$ & $B_{2}$ & $B_{3}$ & $B_{3}$ & $B_{2}$ & $B_{3}$ & $B_{3}$ \\
\hline \multicolumn{3}{|c|}{$A_{3}$} & \multicolumn{3}{|c|}{$A_{1}$} & \multicolumn{3}{|c|}{$A_{3}$} & \multicolumn{3}{|c|}{$A_{2}$} \\
\hline$B_{2}$ & $B_{3}$ & $B_{3}$ & $B_{2}$ & $B_{3}$ & $B_{3}$ & $B_{2}$ & $B_{3}$ & $B_{3}$ & $B_{2}$ & $B_{3}$ & $B_{3}$ \\
\hline \multicolumn{3}{|c|}{$\bar{~} \overline{A_{3}}$} & \multicolumn{3}{|c|}{$A_{2}$} & \multicolumn{3}{|c|}{$A_{1}$} & \multicolumn{3}{|c|}{$A_{3}$} \\
\hline$B_{2}$ & $B_{3}$ & $B_{3}$ & $B_{2}$ & $B_{3}$ & $B_{3}$ & $B_{2}$ & $B_{3}$ & $B_{3}$ & $B_{2}$ & $B_{3}$ & $B_{3}$ \\
\hline \multirow{2}{*}{\multicolumn{3}{|c|}{$A_{1}$}} & \multirow{2}{*}{\multicolumn{3}{|c|}{$\begin{array}{l}A_{3} \\
B_{3}\end{array}$}} & \multirow{2}{*}{\multicolumn{3}{|c|}{$\begin{array}{l}A_{2} \\
B_{3}\end{array}$}} & \multicolumn{3}{|c|}{$A_{3}$} \\
\hline$B_{2}$ & & & & & & & & & $B_{2}$ & $B_{3}$ & $B_{3}$ \\
\hline
\end{tabular}

Superblock II

\begin{tabular}{|c|c|c|c|c|c|c|c|c|c|c|}
\hline \multicolumn{3}{|c|}{$A_{2}$} & \multicolumn{3}{|c|}{$A_{3}$} & \multicolumn{3}{|c|}{$A_{3}$} & \multicolumn{2}{|l|}{$A_{1}$} \\
\hline$B_{1}$ & $B_{2}$ & $B_{3}$ & $B_{1}$ & $B_{2}$ & $B_{3}$ & $B_{1}$ & $B_{2}$ & $B_{3}$ & \begin{tabular}{l|l}
$B_{1}$ & $B_{2}$ \\
\end{tabular} & $B_{3}$ \\
\hline \multicolumn{3}{|c|}{$A_{3}$} & \multicolumn{3}{|c|}{$A_{1}$} & \multicolumn{3}{|c|}{$\overline{A_{3}}$} & \multicolumn{2}{|l|}{$A_{2}$} \\
\hline$B_{1}$ & $B_{2}$ & $B_{3}$ & $B_{1}$ & $B_{2}$ & $B_{3}$ & $B_{1}$ & $B_{2}$ & $B_{3}$ & \begin{tabular}{l|l}
$B_{1}$ & $B_{2}$ \\
\end{tabular} & $B_{3}$ \\
\hline \multicolumn{3}{|c|}{$A_{3}$} & \multicolumn{3}{|c|}{$A_{2}$} & \multicolumn{3}{|c|}{$A_{1}$} & \multicolumn{2}{|l|}{$A_{3}$} \\
\hline$B_{1}$ & $B_{2}$ & $B_{3}$ & $B_{1}$ & $B_{2}$ & $B_{3}$ & $B_{1}$ & $B_{2}$ & $B_{3}$ & \begin{tabular}{l|l}
$B_{1}$ & $B_{2}$ \\
\end{tabular} & $B_{3}$ \\
\hline & \multicolumn{3}{|c|}{$A_{3}$} & \multicolumn{3}{|c|}{$\bar{~} A_{2}$} & \multicolumn{2}{|l|}{$A_{3}$} \\
\hline$B_{1}$ & $B_{2}$ & $B_{3}$ & $B_{1}$ & $B_{2}$ & $B_{3}$ & $B_{1}$ & $B_{2}$ & $B_{3}$ & \begin{tabular}{l|l}
$B_{1}$ & $B_{2}$ \\
\end{tabular} & $B_{3}$ \\
\hline
\end{tabular}

Superblock III

\begin{tabular}{|c|c|c|c|c|c|c|c|c|c|c|c|}
\hline \multicolumn{3}{|c|}{$A_{2}$} & \multicolumn{3}{|c|}{$A_{3}$} & \multicolumn{3}{|c|}{$A_{3}$} & \multicolumn{3}{|c|}{$A_{1}$} \\
\hline$B_{1}$ & $B_{3}$ & $B_{3}$ & $B_{1}$ & $B_{3}$ & $B_{3}$ & $B_{1}$ & $B_{3}$ & $B_{3}$ & $B_{1}$ & $B_{3}$ & $B_{3}$ \\
\hline \multicolumn{3}{|c|}{$A_{3}$} & \multicolumn{3}{|c|}{$A_{1}$} & \multicolumn{3}{|c|}{$A_{3}$} & \multicolumn{3}{|c|}{$A_{2}$} \\
\hline$B_{1}$ & $B_{3}$ & $B_{3}$ & $B_{1}$ & $B_{3}$ & $B_{3}$ & $B_{1}$ & $B_{3}$ & $B_{3}$ & $B_{1}$ & $B_{3}$ & $B_{3}$ \\
\hline \multicolumn{3}{|c|}{$A_{3}$} & \multicolumn{3}{|c|}{$A_{2}$} & \multicolumn{3}{|c|}{$A_{1}$} & \multicolumn{3}{|c|}{$A_{3}$} \\
\hline$B_{1}$ & $B_{3}$ & $B_{3}$ & $B_{1}$ & $B_{3}$ & $B_{3}$ & $B_{1}$ & $B_{3}$ & $B_{3}$ & $B_{1}$ & $B_{3}$ & $B_{3}$ \\
\hline \multicolumn{3}{|c|}{$A_{1}$} & \multicolumn{3}{|c|}{$A_{3}$} & \multicolumn{3}{|c|}{$A_{2}$} & \multicolumn{3}{|c|}{$A_{3}$} \\
\hline$B_{1}$ & $B_{3}$ & $B_{3}$ & $B_{1}$ & $B_{3}$ & $B_{3}$ & $B_{1}$ & $B_{3}$ & $B_{3}$ & $B_{1}$ & $B_{3}$ & $B_{3}$ \\
\hline
\end{tabular}

Superblock IV

\begin{tabular}{|c|c|c|c|c|c|c|c|c|c|c|c|}
\hline \multicolumn{3}{|c|}{$A_{2}$} & \multicolumn{3}{|c|}{$A_{3}$} & \multicolumn{3}{|c|}{$A_{3}$} & \multicolumn{3}{|c|}{$A_{1}$} \\
\hline$B_{1}$ & $B_{2}$ & $B_{3}$ & $B_{1}$ & $B_{2}$ & $B_{3}$ & $B_{1}$ & $B_{2}$ & $B_{3}$ & $B_{1}$ & $B_{2}$ & $B_{3}$ \\
\hline \multicolumn{3}{|c|}{$A_{3}$} & \multicolumn{3}{|c|}{$A_{1}$} & \multicolumn{3}{|c|}{$A_{3}$} & \multicolumn{3}{|c|}{$A_{2}$} \\
\hline$B_{1}$ & $B_{2}$ & $B_{3}$ & $B_{1}$ & $B_{2}$ & $B_{3}$ & $B_{1}$ & $B_{2}$ & $B_{3}$ & $B_{1}$ & $B_{2}$ & $B_{3}$ \\
\hline \multicolumn{3}{|c|}{$A_{3}$} & \multicolumn{3}{|c|}{$A_{2}$} & \multicolumn{3}{|c|}{$A_{1}$} & \multicolumn{3}{|c|}{$A_{3}$} \\
\hline$B_{1}$ & $B_{2}$ & $B_{3}$ & $B_{1}$ & $B_{2}$ & $B_{3}$ & $B_{1}$ & $B_{2}$ & $B_{3}$ & $B_{1}$ & $B_{2}$ & $B_{3}$ \\
\hline & & & \multicolumn{3}{|c|}{$A_{3}$} \\
\hline$B_{1}$ & $B_{2}$ & $B_{3}$ & $B_{1}$ & $B_{2}$ & $B_{3}$ & $B_{1}$ & $B_{2}$ & $B_{3}$ & $B_{1}$ & $B_{2}$ & $B_{3}$ \\
\hline
\end{tabular}

Figure 1. Arrangement of the whole plot and the subplot treatments in the superblocks 
Table 6. Stratum efficiency factors in the example

\begin{tabular}{|c|c|c|c|c|c|c|}
\hline \multirow{2}{*}{ Type of contrasts } & \multirow{2}{*}{$\begin{array}{l}\text { Number } \\
\text { of contrasts }\end{array}$} & \multicolumn{5}{|c|}{ Strata } \\
\hline & & I & II & III & IV & $\mathrm{V}$ \\
\hline$A^{(1)}$ & 1 & 0 & 0 & 0 & 1 & 0 \\
\hline$A^{(2)}$ & 1 & 0 & 0 & 0 & 1 & 0 \\
\hline$B^{(1)}$ & 1 & $1 / 9$ & 0 & 0 & 0 & $8 / 9$ \\
\hline$B^{(2)}$ & 1 & $1 / 9$ & 0 & 0 & 0 & $8 / 9$ \\
\hline$A^{(1)} \times B^{(1)}$ & 1 & 0 & 0 & 0 & $1 / 9$ & $8 / 9$ \\
\hline$A^{(1)} \times B^{(2)}$ & 1 & 0 & 0 & 0 & $1 / 9$ & $8 / 9$ \\
\hline$A^{(2)} \times B^{(1)}$ & 1 & 0 & 0 & 0 & $1 / 9$ & $8 / 9$ \\
\hline$A^{(2)} \times B^{(2)}$ & 1 & 0 & 0 & 0 & $1 / 9$ & $8 / 9$ \\
\hline
\end{tabular}

basic contrasts as $A^{(1)}$ and $A^{(2)}$ for the subplot treatments. $A^{(i)} \times B^{(j)}$, $i, j=1,2$, denote the basic contrasts among the interaction effects of the corresponding whole plot and subplot treatments; for example, $A^{(1)} \times B^{(1)}$ denotes the basic contrast among the interaction effects of the whole plot and subplot treatments.

\section{Final remarks}

This paper has presented a survey of the design of experiments with split units in which whole plot treatments are arranged in a Latin square, modified Latin square or Youden square. The subplot treatments can be arranged in any proper block design. In particular we can find a design for the subplot treatments satisfying all of the experimenter's suggestions concerning statistical properties, especially with regard to efficiency. The treatment merging method can be useful in searching for proper optimal designs. Moreover, the problem of introducing whole plot control treatments or subplot control treatments is examined.

The problems examined in the paper demonstrate the considerable potential of the designs considered as regards their optimal use in practice. 


\section{Acknowledgements}

The authors would like to thank JSPS and PAN (Polish Academy of Sciences) for giving them the opportunity to carry out this joint research in Japan and in Poland. The research was partially supported by JSPS, Grant-in-Aid for Scientific Research (C) 24540139 and Grant-in-Aid for Young Scientists (B) 25730020 .

\section{REFERENCES}

Bailey R. (1995): General balance: artificial theory or practical relevance. Proc. of the International Conference on Linear Statistical Inference, LINSTAT'93, Mathematics and Applications. Vol. 306, Kluwer Academic Publishers, Dordrecht: 171-184.

Caliński T., Kageyama S. (2000): Block Designs: Randomizatiom Approach, Volume I: Analysis, Lecture Notes in Statistics, 150, Springer-Verlag, New York.

Caliński T., Kageyama S. (2003): Block Designs: Randomizatiom Approach, Volume II: Design, Lecture Notes in Statistics, 170, Springer-Verlag, New York.

Ceranka B., Mejza S. (1987): Merging of treatments in certain partially efficiency balanced block designs with two different numbers of replications. Calcutta Statist. Assoc. Bull. 36: 49-55.

Clatworthy W.H. (1973): Tables of Two-Associate-Class Partially Balanced Designs. NBS Applied Mathematics Series 63. Washington, D.C, USA.

Hering F., Mejza S. (2002): An incomplete split-block design generated by GDPBIBD(2)s. Journal of Statistical Planning and Inference 106: 125-134.

Houtman A.M., Speed T.P. (1983): Balance in designed experiments with orthogonal block structure. Ann. Statist., 11: 1069-1085.

Kachlicka D., Hering F., Mejza S. (2004): Control treatments in Youden Square with Split Units. Folia Fac. Sci. Nat. Univ. Masaryk. Brunensis, Mathematica 15: 137-144.

Kachlicka D., Mejza S. (1996): Repeated row-column designs with split units. Comp. Statist. \& Data Analysis 21: 293-305.

Kachlicka D., Mejza S. (2003): Whole plot control treatments in Youden square with split units. Colloquium Biometryczne 33a: 77-84.

Kachlicka D., Mejza S. (2006): Repeated Youden Square with Split Units generated by GDPBIBD(2). XVIIth. Summer School of Biometrics, "Current Trends in Biometrical Research", G. J. Mendel University of Agriculture and Forestry, Lednice, August 21 - 25. 2006. Eds. J. Hartmann i J. Michalek: 159-160.

Kuriki S., Mejza S., Mejza I., Kachlicka D. (2009): Repeated Youden squares with subplot treatments in a proper incomplete block design. Biometrical Letters 46(2): 153-162.

Mejza I., Mejza S. (1996): Incomplete split plot designs generated by $\operatorname{GDPBIBD}(2)$. Calcutta Statist. Assoc. Bull. 46: 117-127. 
Mejza S. (1992): On some aspects of general balance in designed experiments. Statistica, anno LII, 2: 263-278.

Mejza S., Kuriki S. (2013): Youden Square with Split Units. J.L. da Silva et al. (eds.), Advances in Regression, Survival Analysis, Extreme values, Markov Processes and Other Statistical Applications, Studies in Theoretical and Applied Statistics, DOI 10.1007/978-3-642-34904-1 1; Springer-Verlag Berlin Heidelberg: 3-10.

Mejza S., Kuriki S. Kachlicka D. (2009). Repeated Youden Squares with subplot treatments in a group-divisible design. Journal of Statistics and Applications 4: 369-377.

Pearce S.C., Caliński T., Marshall T.F. de C. (1974): The basic contrasts of an experimental design with special reference to the analysis of data. Biometrika 61: 449-460. 